\section{LEF1-mediated regulation of Delta-like1 links Wnt and Notch signaling in somitogenesis}

\author{
Juan Galceran, ${ }^{1,3}$ Claudio Sustmann, ${ }^{1,2}$ \\ Shu-Chi Hsu, ${ }^{1}$ Stephanie Folberth, ${ }^{1,2}$ and \\ Rudolf Grosschedl ${ }^{1,2,4}$
}

${ }^{1}$ Gene Center and Institute of Biochemistry, University of Munich, 81377 Munich, Germany; ${ }^{2}$ Max-Planck-Institute of Immunobiology, 79108 Freiburg, Germany

Wnt signaling, which is mediated by LEF1/TCF transcription factors, has been placed upstream of the Notch pathway in vertebrate somitogenesis. Here, we examine the molecular basis for this presumed hierarchy and show that a targeted mutation of Lef1, which abrogates LEF1 function and impairs the activity of coexpressed TCF factors, affects the patterning of somites and the expression of components of the Notch pathway. LEF1 was found to bind multiple sites in the Dll1 promoter in vitro and in vivo. Moreover, mutations of LEF1-binding sites in the Dll1 promoter impair expression of a Dl11$L a c Z$ transgene in the presomitic mesoderm. Finally, the induced expression of LEF1- $\beta$-catenin activates the expression of endogenous $D 111$ in fibroblastic cells. Thus, Wnt signaling can affect the Notch pathway by a LEF1mediated regulation of $D l 11$.

Supplemental material is available at http://

www.genesdev.org.

Received August 11, 2004; revised version accepted September 28, 2004.

Vertebrate somitogenesis generates segmental body structures, such as vertebrae, spinal nerves, axial muscles, and blood vessels. Somites are blocks of mesodermal cells that are generated from the unsegmented paraxial mesoderm, termed presomitic mesoderm (PSM), at the tail end of the embryo. After somites are cleaved off at the anterior end of the PSM, somitic cells differentiate into various axial structures, depending on their relative position. Cells near the ectoderm differentiate into the dermomyotome, whereas medial cells differentiate into the sclerotome. In addition, newly formed somites have rostral and caudal compartments that allow for the subsequent resegmentation process of the sclerotome (for reviews, see Pourquié 2001; Saga and Takeda 2001).

The segmental structure of somites is generated in a

[Keywords: Somitogenesis; LEF1/TCF; Wnt; Notch; Dll1]

${ }^{3}$ Present address: Instituto de Neurociencias, Campus San Juan, 03550

Sant Joan d'Alacant, Spain

${ }^{4}$ Corresponding author.

E-MAIL grosschedl@immunbio.mpg.de; FAX 49-761-5108-799.

Article and publication are at http://www.genesdev.org/cgi/doi/10.1101/ gad.1249504 spatially periodic pattern that is determined by a temporal oscillation of the expression of genes (for review, see Pourquié 2003). In vertebrates, genes of the Notch-Delta signaling pathway, including the Notch-responsive HES transcription factors, the Notch ligand Delta-like (Dll), and the Notch antagonist lunatic fringe $(\operatorname{Lnfg})$, have been found to cycle during somitogenesis (for review, see Pourquié 2003). A role of Notch signaling in the rostrocaudal patterning of somites and in the regulation of the oscillations of cycling genes was inferred from gene disruptions of $R B P j \kappa$ and $L n f g$, as well as from transgenic expression of a dominant negative form of delta-like1 (Conlon et al. 1995; Hrabe de Angelis et al. 1997; Evrard et al. 1998; Zhang and Gridley 1998; Cordes et al. 2003). In addition, Wnt signaling has been implicated in the regulation of somitogenesis (Auleha et al. 2003). A reduction of Wnt3a expression in mice carrying a hypomorphic allele of Wnt3a, termed vestigial tail $(v t)$, results in alterations of vertebrate identity and oscillating Notch activity (Greco et al. 1996; Ikeya and Takeda 2001; Auleha et al. 2003). These results and those of Wnt3a misexpression experiments have been interpreted to suggest a hierarchy of Wnt and Notch signaling, in which Wnt3a acts upstream of Notch signaling in the regulation of somitogenesis. However, the molecular link between Wnt signaling and Notch signaling remains unclear.

LEF1/TCF transcription factors have been identified as nuclear mediators of Wnt signaling that activate Wntresponsive genes in association with $\beta$-catenin (for review, see van Noort and Clevers 2002). Lef1 and Tcf1 are coexpressed in the PSM of developing mice, and the targeted inactivation of both genes resulted in the lack of paraxial mesoderm and in the generation of multiple neural tubes, phenotypes that are identical to those of the Wnt3a null mutation (Takada et al. 1994; Yoshikawa et al. 1997; Galceran et al. 1999). In contrast, the individual null mutations of Lef1 and Tcf1 show no apparent defect in mesoderm formation or somite patterning, consistent with a redundant function of the transcription factors in these processes (van Genderen et al. 1994; Verbeek et al. 1995). Therefore, neither the double null mutations nor the single null mutations provided an insight into the presumed role of these transcription factors in somitogenesis and segmental patterning.

\section{Results and Discussion}

In this study, we examined somitogenesis in mice carrying a mutant allele of $L e f 1$, in which the bacterial $\beta$-galactosidase ( $L a C Z$ ) gene had been inserted in-frame into the exon encoding the DNA-binding domain of LEF1. We have previously shown that this targeted mutation allows for the stable accumulation of a truncated form of LEF1 that can interact with $\beta$-catenin but fails to bind DNA (Galceran et al. 2000). Moreover, we found that the truncated LEF1 protein interferes with the $\beta$-catenin-dependent transcriptional activation by coexpressed TCF transcription factors in transfection assays, and we observed that the Lef1- $\beta$ gal mutation generates a more severe phenotype in hippocampus development than the null mutation of Lef1 (Galceran et al. 2000). However, the homozygous Lef1- $\beta g a l$ mutation is not as severe as the Lef1 $1^{-/-}$Tcf1 $1^{-1-}$ mutation and mice carrying these mu- 
tations die perinatally and at embryonic day 10.5 (E10.5), respectively (Galceran et al. 1999, 2000).

Analysis of the skeleton of newborn homozygous mutant Lef1-ßgal mice by Alcian blue/alizarin red staining showed that the vertebral column and the ribcage, derivatives of somites, are severely malformed (Fig. 1a-d). The number of vertebrae was reduced and many vertebrae were fused. The caudal region was shortened and the tail was reduced to a small curly stump. In addition, the mutants showed an overall decrease in the number of ribs, fusions, and bifurcations of the ribs, as well as a severe malformation of the sternum. These data suggest that LEF1/TCF transcription factors are not only involved in the generation, but also in the patterning, of paraxial mesoderm. Consistent with the expression of Lef1-ßgal in the developing limb buds and the early arrest of limb bud development in Lef1 ${ }^{-/-} \mathrm{Tcf1} 1^{-/-}$embryos (Fig. 2A; Galceran et al. 1999), the forelimbs and hindlimbs were severely malformed in homozygous Lef1Bgal mice, with a consistent reduction in the number of fingers (Fig. 1e,f; data not shown).

To examine the potential role of LEF1/TCF factors in somitogenesis, we analyzed the expression of the Lef1Bgal gene and the morphology of the mutant mice at E9.5 (Fig. 2A). Whole-mount staining for LacZ activity in heterozygous Lef1- $\beta g a l$ mice revealed expression in the PSM, the newly formed somites, and the forelimb buds (Fig. 2A, panel a), consistent with the previously described pattern of Lef1 mRNA expression (Oosterwegel et al. 1993; Galceran et al. 1999). Homozygous mutant Lef1-ßgal mice showed a severely deformed tail and a lack of segmented somites (Fig. 2A, panels a-d). However, homozygous Lef1-ßgal embryos have no extra neural tubes, which are formed at the expense of paraxial mesoderm in both $L e f 1^{-/-} \mathrm{Tcf1} 1^{-/-}$and Wnt $3 \mathrm{a}^{-/-}$embryos

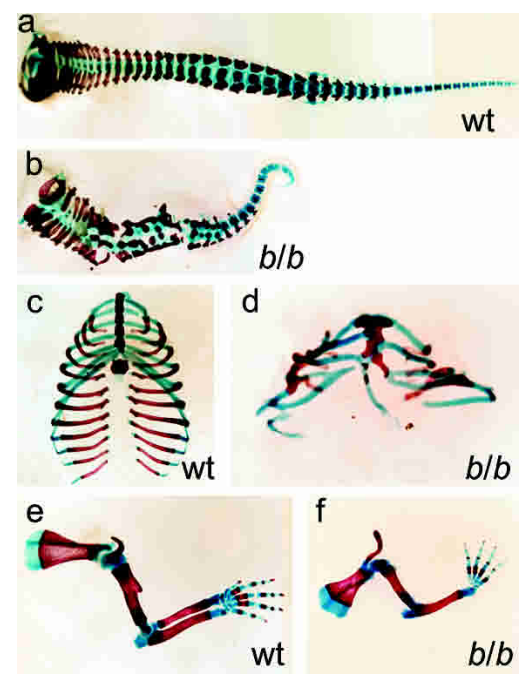

Figure 1. Skeletal malformations in homozygous Lef1- $\beta g a l$ mice. Alcian blue/alizarin red staining of skeletal preparations of wildtype $(\mathrm{wt} ; a, c, e)$ and homozygous Lef1- $\beta g a l(b / b ; b, d, f)$ newborns. $(a, b)$ The vertebral column of $b / b$ mutants shows a reduction in the number of vertebrae with extensive fusions in their bodies and neural arches. $(c, d)$ The ribcage of mutant mice contains fewer ribs that show fusions, bifurcations, and asymmetric attachment to the sternum. $(e, f)$ The forelimbs of homozygous Lef1- $\beta g a l$ newborns are malformed, with a frequent loss of the radius and a consistent reduction in the number of fingers.

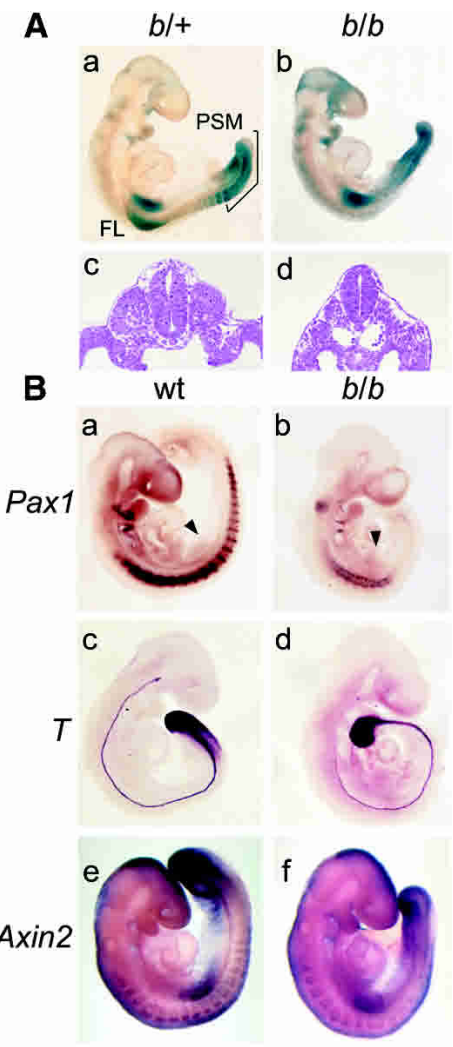

Figure 2. Defects of somite formation in homozygous Lef1-ßgal mice. (A) Analysis of Lef1- $\beta g a l$ expression in heterozygous $(b /+)$ and homozygous $(b / b)$ E9.5 embryos by whole-mount staining for LacZ activity. (Panels $a, b)$ Abundant Lef1- $\beta g a l$ expression is observed in forelimb buds (FL), the presomitic mesoderm (PSM), and newly formed somites. (Panels $c, d$ ) Cross-sections of the thoracic region show the lack of somites in the mutant embryos. $(B)$ Expression of molecular markers in E9.5 wild-type (wt) and homozygous Lef1- $\beta g a l$ embryos by whole-mount in situ hybridization. (Panels $a, b$ ) In mutants, the segmented expression pattern of the sclerotome marker Pax1 is severely impaired. (Panels $c, d)$ Brachyury $(T)$ expression in the anterior region of the PSM is reduced in mutant embryos $(b / b)$, but normal expression is detected in the posterior region of the PSM and notochord. (Panels e,f) Expression of Axin2 in the PSM is reduced in mutant embryos. Arrowheads point to the forelimb buds.

(Takada et al. 1994; Yoshikawa et al. 1997; Galceran et al. 1999).

To further define these defects, we analyzed the expression of molecular markers that identify specific mesodermal cell populations and structures (Fig. 2B). Expression of Pax1, which is normally expressed in ventromedial cells of the sclerotome, is severely impaired in homozygous Lef1- $\beta g a l$ mice in the region caudal of the forelimbs (Fig. 2B, panels a,b). As some thoracic skeletal elements are formed in the mutant mice, these data suggest that the formation of sclerotome is delayed and/or reduced. Likewise, expression of Pax3 showed a markedly reduced mesodermal expression in the mutant embryos (Supplementary Fig. 1c,d). We also examined the expression of two genes, Brachyury $(T)$ and Axin2, that have been identified as direct targets for LEF1 and Wnt signaling (Yamaguchi et al. 1999; Galceran et al. 2001; Auleha et al. 2003). The expression of $T$ was reduced in the anterior region of the PSM of homozygous Lef1- $\beta g a l$ mice, in contrast to the complete lack of expression of $T$ in Lef1 ${ }^{-/-}$Tcf1 ${ }^{-/-}$mice (Fig. 2B, panels c,d; Galceran et al. 
2001). The expression of Axin2 was more severely affected in the PSM of homozygous Lef1- $\beta g a l$ mice (Fig. $2 \mathrm{~B}$, panels e,f), whereas the expression of Wnt3a was normal (Supplementary Fig. 1e,f).

To examine the role of LEF1/TCF factors in the patterning of somites, we analyzed the developing peripheral nervous system in Lef1- $\beta g a l$ mice at E11.5 by antineurofilament staining. Mutant mice showed a lack or misrouting of neural crest-derived spinal nerves that traverse the anterior compartment of somites in wild-type embryos (Fig. 3A, panels a,b; Supplementary Fig. 1g,h).

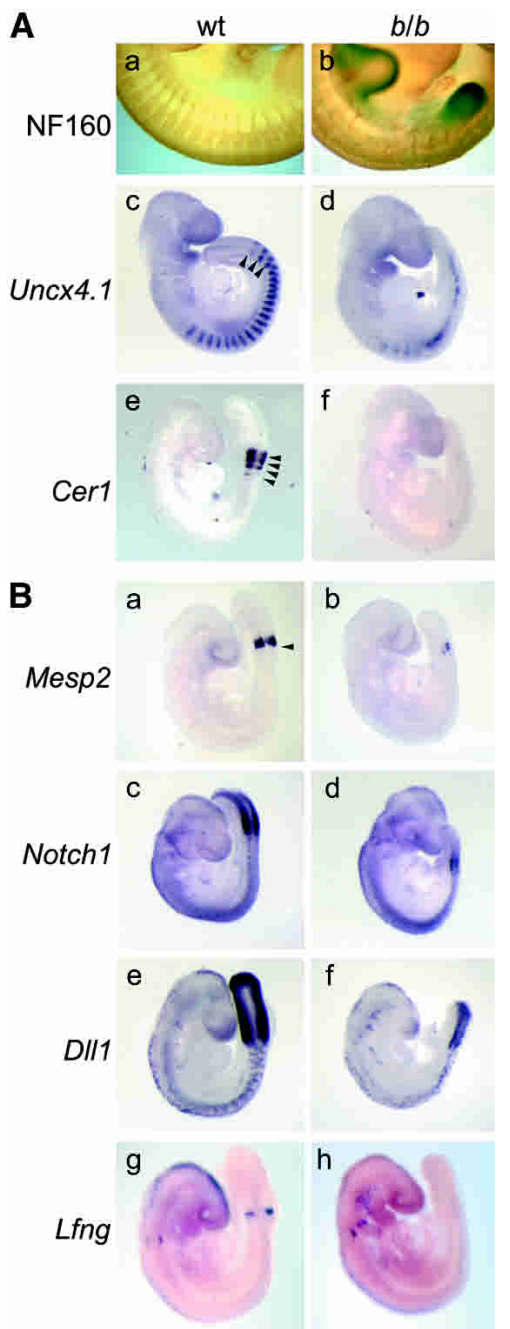

Figure 3. (A) Defects in the patterning and polarity of somites in homozygous Lef1- $\beta g a l(b / b)$ mice. (Panels $a, b)$ Whole-mount antineurofilament staining of E11.5 embryos shows the lack of regularly spaced spinal nerve projections and a misrouting of axons in mutant embryos. (Panels $c-f$ ) Whole-mount in situ hybridizations of E9.5 mutant embryos show a caudal loss of expression of the posterior somite marker Uncx4.1 and the abrogation of expression of the anterior somite marker Cerberus (Cer1). Arrowheads indicate somite boundaries. $(B)$ Expression of Notch signaling components in wildtype and homozygous Lef1- $\beta g a l$ mice. (Panels $a, b$ ) Expression of Mesp2 in the anterior presomitic mesoderm (PSM) is markedly reduced in mutant embryos. Arrowhead indicates the anterior boundary of the PSM. (Panels c-f) Expression of Notch1 and Dll1 in the PSM is also reduced in mutant embryos but is maintained in neural tissues. (Panels $g, h)$. Expression of the Notch antagonist Lnfg is undetectable in the newly formed somites of mutant embryos.
Moreover, the mutant mice showed fusions of somites and a lack of expression of Semaphorin D, which is expressed normally in the posterior half of the dermomyotome (Supplementary Fig. 1k,1; Adams et al. 1996). With the aim of obtaining additional evidence for a defect in the patterning of somites, we analyzed the expression of the paired-type homeobox gene Uncx4.1, which is restricted to the posterior somite halves (Neidhardt et al. 1997), and Cerberus, cer1, which is expressed in the anterior halves of newly formed somites (Biben et al. 1998). In homozygous mutant mice, Uncx 4.1 expression can be detected; however, the segmental pattern is abrogated caudal of the forelimb bud and the pattern is impaired in the cervical somites (Fig. 3A, panels c,d). The expression of Cerberus is virtually lost in the mutant mice (Fig. 3A, panel f). Together, these data confirm the defect of homozygous Lef1-ßgal mice in the rostrocaudal patterning of somites.

The Notch signaling pathway has been implicated as a major regulatory determinant in the formation and patterning of somites (for review, see Pourquie 2003). The Mesp2 gene, which encodes a transcription factor that is expressed in the anterior PSM, is important for somite boundary formation and the establishment of rostrocaudal polarity (Saga et al. 1997). Mesp2 antagonizes the expression of Uncx4.1 and is involved in a complex feedback regulation with Dll1 (Takahashi et al. 2003). In addition, Notch1 and Dll1 are important for the rostrocaudal polarity and the oscillation of cycling genes (for review, see Pourquié 2003). Expression analysis of these genes in homozygous Lef1- $\beta g a l$ mice showed a marked reduction in the expression of Mesp2, Notch1, and Dll1 (Fig. 3B, panels a-f). In addition, we observe a decrease in the expression of $\mathrm{Lnfg}$ in the newly formed somites of mutant embryos (Fig. 3B, panels g,h). The reduction of the expression of multiple markers of rostrocaudal polarity could be interpreted to suggest that LEF1/TCF proteins regulate several genes in the Notch/Delta/Mesp2 pathway and/or that a LEF1/TCF-regulated gene is involved in genetic interactions with other components of the pathway. We favor the first view because the phenotype of the homozygous Lef1- $\beta g a l$ mice differs from

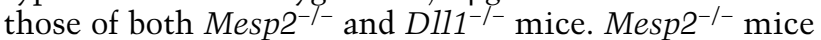
show an expansion of Dll1 expression and a caudalization of somites, (Saga et al. 1997), whereas Dll1-null embryos show a rostralization of somites (del Barco Barrantes et al. 1999). In contrast, the homozygous Lef1Bgal mice show defects in both patterning events.

The marked decrease in the expression of Notch1 and Dll1 in homozygous Lef1- $\beta g a l$ mice is consistent with the proposed hierarchy of the Wnt and Notch signaling pathways, and this raised the possibility that components of the Notch pathway are directly regulated by LEF1/TCF factors. To this end, we examined the promoter regions of these genes for the presence of potential LEF1/TCF-binding sites. The 5'-flanking regions of both Notch1 and D111 genes were found to contain multiple potential LEF1/TCF-binding sites (Fig. 4A; data not shown). Previous transgenic analysis of the transcriptional regulatory sequences of the Dll1 gene identified distinct elements that direct gene expression in the paraxial mesoderm and nervous system (Beckers et al. 2000). Two sequence homology domains (HDs) were found to confer neuronal expression, whereas a $1.5-\mathrm{kb}$ region that resides between the HDs acts as a mesodermspecific enhancer. In addition, a $1.6-\mathrm{kb}$ region that in- 
A

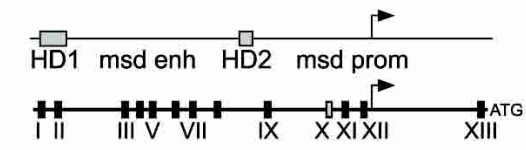

B

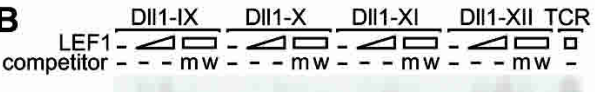

LEF1:DNA

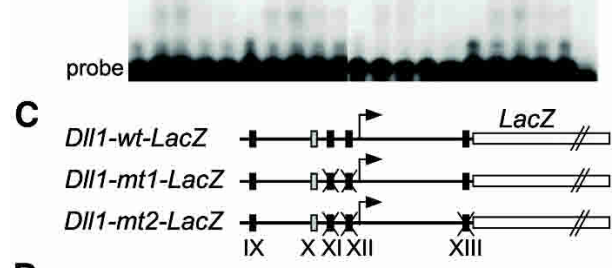

D
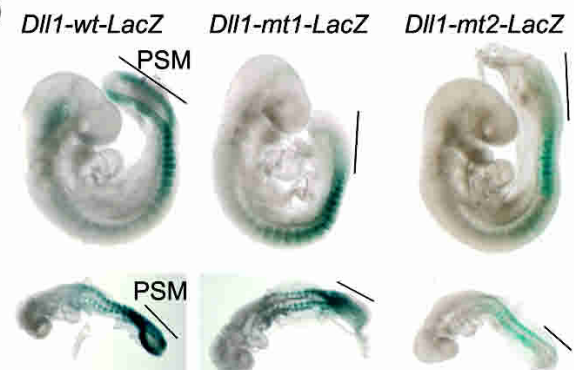

E
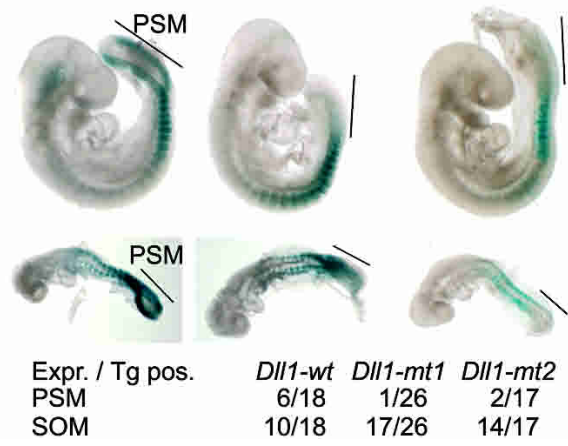

Figure 4. LEF1-mediated regulation of Dll1. (A) Schematic presentation of the $5^{\prime}$-flanking region of Dll1, including homology domain (HD)-1 and HD-2, the mesoderm enhancer (msd enh), and promoter (msd prom). The positions of confirmed LEF1/TCF-binding sites are indicated by boxes. Black and gray boxes represent strong and weak binding sites, respectively. $(B)$ Electrophoretic mobility shift assay showing binding of recombinant LEF1 to sites in the msd promoter region. Purified LEF1 (100 or $300 \mathrm{ng}$ ) was incubated with radiolabeled oligonucleotides encompassing binding sites IX-XII. The specificity of binding was confirmed by addition of 100-fold molar excess of unlabeled oligonucleotides encompassing a wild-type $(\mathrm{w})$ or mutated $(\mathrm{m})$ LEF1 consensus site from the TCR $\alpha$ enhancer (Galceran et al. 2001). (C) Schematic presentation of Dll1-LacZ gene constructs containing the msd promoter, in which the positions of wild-type or mutated LEF1-binding sites are indicated. $(D)$ Wholemount staining for LacZ activity of representative E9.5 (top) and E8.5 (bottom) embryos carrying wild-type or mutant transgenes. The wild-type transgene is expressed in the PSM (bar) and somites, whereas the mutant transgenes are expressed only in the somites. $(E)$ Quantitation of the transgenic expression analysis, in which the frequency of expression in PSM and somites (SOM) is presented as the ratio of number of embryos that express (Expr.)/carry a transgene (TG pos.).

cludes the transcription start site directs expression in the paraxial mesoderm and in developing somites. The LEF1-binding sites in Dll1 were confirmed as bona fide binding sites by electrophoretic mobility shift assays, using recombinant LEF1 protein and radiolabeled oligonucleotides (Fig. 4B; Supplementary Fig. 2).

The functional importance of the LEF1-binding sites was determined by generating transgenic mice with Dll1 promoter gene constructs, in which wild-type or mutated Dll1 promoter fragments were linked to the bacterial $\beta$-galactosidase ( $\mathrm{LaCZ}$ ) gene (Fig. $4 \mathrm{C}$ ). Six out of 18 transgenic embryos expressed the Dl11-wt-LacZ gene in the PSM and somites (Fig. 4D,E). In contrast, the expres- sion of mutant transgenes carrying point mutations in two or three LEF1-binding sites was markedly impaired in the PSM (Fig. 4D,E). Only $1 / 26$ and 2/17 embryos carrying the Dll1-mt1-LacZ and Dll1-mt2-LacZ transgenes, respectively, showed expression in the PSM. However, the expression of the mutant transgenes in the somites was similar to that of the wild-type transgene. These data indicate that the activity of the Dll1 promoter in the PSM, but not in the somites, is dependent on the LEF1-binding sites.

To obtain further evidence for the regulation of Dll1 by LEF1, we examined whether the induced expression of a LEF1- $\beta$-catenin fusion protein, in which the C-terminal activation domain of $\beta$-catenin is linked to the $\mathrm{N}$ terminus of LEF1 (Fig. 5A; Hsu et al. 1998), results in the

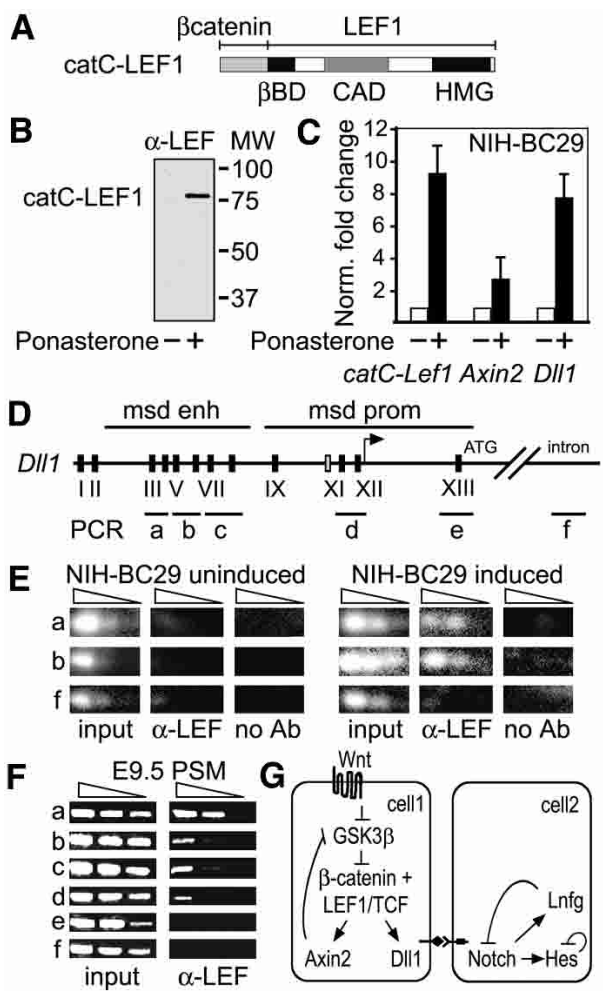

Figure 5. catC-LEF-induced expression of Dl11 and binding of LEF1 to Dll1 regulatory sequences in vivo. (A) Schematic presentation of the catC-LEF1 protein that activates gene expression in the absence of Wnt signals (Hsu et al. 1998). (B) Anti-LEF-immunoblot analysis of NIH-BC29 cells containing a catC-LEF1 construct under the control of ecdysone-response elements. Protein expression can be detected $12 \mathrm{~h}$ after treatment of the cells with Ponasterone. $(C)$ Quantitative RT-PCR to detect the expression of the endogenous Axin2 and Dll1 genes in BC29 cells, uninduced and induced for $12 \mathrm{~h}$. The levels of expression were normalized against the expression of actin. $(D)$ Schematic presentation of the msd regulatory regions of the Dll1 gene, in which the positions of the LEF1-binding sites and the regions analyzed by chromatin immunoprecipitations (ChIPs) are indicated. (E) ChIP of uninduced and induced BC29 cells to detect binding of catC-LEF1 to Dll1 sequences in vivo. Semiquantitative PCR was performed with serial dilutions of template DNA. Binding can be detected with anti-LEF antibodies, but not without addition of antibodies. (F) ChIP of PSM from E9.5 embryos to detect binding of endogenous LEF1 to Dll1 regulatory sequences in vivo. Binding can be detected in both msd enhancer and promoter regions. $(G)$ Schematic model for the regulation of Dl11 by LEF1, which links the Wnt signaling pathway with the Notch/Dll pathway in somitogenesis. Negative feedback loops that regulate the cycling expression of genes are indicated (for review, see Pourquié 2003). 
expression of endogenous Dll1 in a fibroblastic cell line. We have previously shown that the LEF- $\beta$-catenin fusion protein activates Wnt-response elements in the absence of a Wnt signal (Hsu et al. 1998). We generated a stably transfected cell line (NIH-BC29), in which the catC-Lef1 gene had been linked to ecdysone-response elements. Within $12 \mathrm{~h}$ after treating the cells with Ponasterone (No et al. 1996), catC-LEF protein could be detected by immunoblot analysis (Fig. 5B). Quantitative RT-PCR assays confirmed a 12-fold increase of catC-Lef1 transcripts and showed an eight- and threefold increase in the levels of expression of endogenous Dll1 and Axin2 genes, respectively (Fig. 5C). This increase in Dll1 expression is somewhat modest, given the multiplicity of LEF1-binding sites, and suggests the possibility that the regulation of Dll1 by LEF1 in vivo requires the cooperation with another transcription factor. TBX6 has been shown to participate in the regulation of Dll1 (White et al. 2003), and in an independent study, the labs of Gossler and Herrmann have found that LEF1 and TBX6 collaborate in the regulation of Dll1 (Hofmann et al. 2004).

We also examined whether the binding of LEF1 to the Dll1 regulatory regions can be detected in vivo. Chromatin immunoprecipitations (ChIPs) with anti-LEF antibodies and semiquantitative PCR amplification of immunoprecipitated chromatin fragments with primers flanking the LEF1-binding sites in the msd enhancer of Dll1 indicated that LEF1 is bound to endogenous Dll1 sequences in induced, but not in uninduced, NIH-BC29 cells (Fig. 5D,E). As a control, no binding of LEF1 was detected in an intron region of DI11 and in samples without $\alpha$-LEF1 antibodies. We also examined whether endogenous LEF1 is bound to Dll1 regulatory sequences by performing ChIP experiments with chromatin from PSM of wild-type E9.5 embryos (Fig. 5F). These experiments indicated that LEF1 binds to multiple sites in both enhancer and promoter of Dll1 in vivo.

Taken together, these data suggest that Wnt-responsive transcription factors of the LEF1/TCF family are involved in a direct regulation of Dll1, thus providing a molecular link between the Wnt and Notch signaling pathways in somitogenesis (Fig. 5G). LEF1/TCF proteins have been shown to directly regulate Fgf4 in epithelialmesenchymal interactions during tooth development (Kratochwil et al. 2002), and LEF1 protein has been found to interact and functionally collaborate with BMP/ TGFß-responsive Smad proteins (Labbe et al. 2000; Nishita et al. 2000). Thus, Wnt signaling can interact via LEF1/TCF transcription factors with multiple signaling pathways, which may help to diversify the biological effects of Wnt signaling.

\section{Materials and methods}

Cloning of Dll1-LacZ gene constructs and generation of a cell line with an inducible catC-LEF gene

The region of $D 111$ residing between -1631 and -7 upstream of the translation initiation codon, which includes the mesoderm-specific promoter and transcription start site (Beckers et al. 2000), was obtained by PCR amplification and cloned into phsp68-LacZpA, from which the hsp promoter was removed (Kothary et al. 1989). Oligonucleotide-directed point mutations in LEF1-binding sites were introduced by using the QuikChange Kit (Stratagene).

For the generation of a cell line in which a Wnt-independent form of LEF1 can be induced, NIH-3T3 cells were stably transfected with a regulator vector pVgRXR (Invitrogen) and subsequently with a gene construct, in which the $\mathrm{C}$-terminal activation domain of $\beta$-catenin was fused with the $\mathrm{N}$ terminus of LEF1 (Hsu et al. 1998). The catC-Lef1 gene was linked to ecdysone-responsive elements, allowing for the induction of expression by treatment of the cells with $30 \mu \mathrm{M}$ Ponasterone (No et al. 1996).

Knockout and transgenic mice, skeletal preparation, and whole-mount in situ hybridizations

The construction of the targeted insertion of the bacterial lac $Z$ gene into the genomic Lef1 locus and the generation of Lef1- $\beta g a l$ mice is described in Galceran et al. (2000). Transgenic mice were generated with Dl11$\mathrm{LacZ}$ gene constructs and genotyped by PCR of yolk sac DNA, and the expression of the transgene was analyzed by staining for $\beta$-galactosidase activity in E8.5 and E9.5 embryos. Alcian blue/alizarin red skeletal staining was performed according to standard procedures. Whole-mount in situ hybridizations were performed with digoxigenin-labeled antisense riboprobes as described in Galceran et al. (1999).

Electrophoretic mobility shift assay

DNA-binding assays were performed by an electrophoretic mobility shift assay with purified recombinant LEF1 protein as described (Galceran et al. 2001). The binding reaction contained $100 \mathrm{ng}$ of purified His-6-tagged LEF1 protein, 2 fmol of 5 ' end-labeled double-stranded oligonucleotide, and $10 \mathrm{mg} / \mathrm{mL}$ poly $(\mathrm{dI}-\mathrm{dC})$. The binding reactions were performed in the absence or presence of 100 -fold molar excess of unlabeled competitor DNA. The nucleotide sequences of the probes are in Supplementary Table 1A.

Chromatin immunoprecipitations

NIH-BC29 cells $\left(5 \times 10^{7}\right)$ or PSM from 40 embryos at E9.5 were prepared and fixed $10 \mathrm{~min}$ with $1 \%$ formaldehyde in PBS at $37^{\circ} \mathrm{C}$. Cross-linking was stopped by addition of glycine to a final concentration of $0.125 \mathrm{M}$. Cells were dispersed and nuclei prepared by addition of hypotonic buffer (10 mM Tris-Cl, $10 \mathrm{mM} \mathrm{NaCl}, 0.2 \%$ NP-40). Cross-linked chromatin was sonicated and after reversal of the cross-links, $50 \mu \mathrm{g}$ chromatin was immunoprecipitated with anti-LEF1 antibodies, as described (Frank et al. 2001). Specific Dll1 sequences were detected by semiquantitative PCR with primers flanking LEF1-binding sites or gene-internal regions, using threefold dilutions of template DNA. The amplifications of BC29 template DNA involved 32 cycles, followed by hybridization with radiolabeled oligonucleotides and detection by phosphorimager. DNA from PSM was amplified by 40 cycles and the products were visualized by ethidium bromide staining. The sequences of the primers are provided in Supplementary Table 1B.

\section{Acknowledgments}

We are grateful to Isabel Farinas and Bettina Engist for the help with the preparations of skeletons and presomitic mesoderm of E9.5 embryos, respectively. We thank Benoit Kanzler for the microinjections of transgenes and Peter Gruss, Rudi Balling, and Eddy de Robertis for gifts of cDNA probes. We also thank Bernhard Herrmann and Achim Gossler for gifts of probes, discussions, and sharing results prior to publication. The work was supported by funds from the German Research Foundation (SFB592) to R.G.

\section{References}

Adams, R.H., Betz, H., and Puschel, A.W. 1996. A novel class of murine semaphorins with homology to thrombospondin is differentially expressed during early embryogenesis. Mech. Dev. 57: 33-45.

Aulehla, A., Wehrle, C., Brand-Saberi, B., Kemler, R., Gossler, A., Kanzler, B., and Herrmann, B.G. 2003. Wnt3a plays a major role in the segmentation clock controlling somitogenesis. Dev. Cell 4: 395-406.

Beckers, J., Caron, A., Hrabé de Angelis, M., Hans, S., Campos-Ortega, J.A., and Gossler, A. 2000. Distinct regulatory elements direct Deltal expression in the nervous system and paraxial mesoderm of transgenic mice. Mech. Dev. 95: 23-34.

Biben, C., Stanley, E., Fabri, L., Kotecha, S., Rhinn, M., Drinkwater, C., Lab, M., Wang, C.C. Nash, A., Hilton, D., et al. 1998. Murine cerberus homologue mCer-1: A candidate anterior patterning molecule. Dev. Biol. 194: 135-151.

Conlon, R.A., Reaume, A.G., and Rossant, J. 1995. Notch1 is required for the coordinate segmentation of somites. Development 121: 15331545. 
Cordes, R., Schuster-Gossler, K., Serth, K., and Gossler, A. 2003. Specification of vertebral identity is coupled to Notch signaling and the segmentation clock. Development 131: 1221-1233.

del Barco Barrantes, I., Elia, A.J., Wunsch, K., Hrabe de Angelis, M., Mak, T.W., Rossant, R., Conlon, R.A., Gossler, A., and de la Pompa, J.L. 1999. Interaction between L-fringe and Notch signaling in the regulation of boundary formation and posterior identity in the presomitic mesoderm of the mouse. Curr. Biol. 9: 470-480.

Evrard, Y.A., Luri, Y., Aulehla, A., Gan, L., and Johnson, R.L. 1998. Lunatic fringe is an essential mediator of somite segmentation and patterning. Nature 394: 377-381.

Frank, S.R., Schroeder, M., Fernandez, P., Taubert, S. and Amati, B. 2001 Binding of c-Myc to chromatin mediates mitogen-induced acetylation of histone H4 and gene activation. Genes \& Dev. 15: 2069-2082.

Galceran, J., Farifias, I., Depew, M.J., Clevers, H., and Grosschedl, R. 1999. Wnt3a $\mathrm{a}^{-/-}$-like phenotype and limb deficiency in Lef $1^{-/-} \mathrm{Tef}^{-/-}$ mice. Genes \& Dev. 13: 709-717.

Galceran, J., Miyashita-Lin, E.M., Devaney, E., Rubenstein, J.L.R., and Grosschedl, R. 2000. Hippocampus development and generation of dentate gyrus granule cells is regulated by LEF-1. Development 127: 469-482.

Galceran, J., Hsu, S.-C., and Grosschedl, R. 2001. Rescue of a Wnt mutation by an activated form of LEF-1: Regulation of maintenance but not initiation of Brachyury expression. Proc. Natl. Acad. Sci. 98: 8668-8673.

Greco, T.L., Takada, S., Newhouse, M.M., McMahon, J.A., McMahon, A.P., and Camper, S.A. 1996. Analysis of the vestigial tail mutation demonstrates that Wnt-3a gene dosage regulates mouse axial development. Genes \& Dev. 10: 313-324.

Hofmann, M., Schuster-Gossler, K., Watabe-Rudolph, M., Aulehla, A., Herrmann, B.G., and Gossler, A. 2004. WNT signaling, in synergy with T/TBX6, controls Notch signaling by regulating Dll1 expression in the presomitic mesoderm of mouse embryos. Genes \& Dev. (this issue).

Hrabe de Angelis, M., Mc Intyre, J., and Gossler, A. 1997. Maintenance of somite borders in mice requires the Delta homologue Dll1. Nature 386: $717-721$.

Hsu, S.-C., Galceran, J., and Grosschedl, R. 1998. Modulation of transcriptional regulation by LEF-1 in response to Wnt-1 signaling and association with $\beta$-catenin. Mol. Cell. Biol. 18: 4807-4818.

Ikeya, M. and Takada, S. 2001. Wnt3a is required for somite specification along the anterioposterior axis of the mouse embryo and for the regulation of cdx-1 expression. Mech. Dev. 103: 27-33.

Kothary, R., Clapoff, S., Darling, S., Perry, M. D., Moran, L.A., and Rossant, J. 1989. Inducible expression of an hsp68-lacZ hybrid gene in transgenic mice. Development 105: 707-714.

Kratochwil, K., Galceran, J., Tontsch, S., Roth, W., and Grosschedl, R. 2002. FGF4, a direct target of LEF1 and Wnt signaling, can rescue the arrest of tooth organogenesis in Lef1/mice. Genes \& Dev. 16: 3173 3185.

Labbe, E., Letamendia, A., and Attisano, L. 2000. Association of Smads with lymphoid enhancer binding factor $1 / \mathrm{T}$ cell-specific factor mediates cooperative signaling by the transforming growth factor- $\beta$ and wnt pathways. Proc. Natl. Acad. Sci. 97: 8358-8363.

Neidhardt, L., Kispert, A., and Herrmann, B. 1997. A mouse gene of the paired-related homeobox class expressed in the caudal somite compartment and in the developing vertebral column, kidney and nervous system. Dev. Genes Evol. 207: 330-339.

Nishita, M., Hashimoto, M.K., Ogata, S., Laurent, M.N., Ueno, N., Shibuya, H., and Cho, K.W. 2000. Interaction between Wnt and TGF- $\beta$ signalling pathways during formation of Spemann's organizer. Nature 403: 781-785.

No, D., Yao, T.P., and Evans, R.M. 1996. Ecdysone-inducible gene expression in mammalian cells and transgenic mice. Proc. Natl. Acad. Sci. 93: 3346-3351.

Oosterwegel, M., van de Wetering, M., Timmerman, J., Kruisbeek, A., Destree, O., Meijlink, F., and Clevers, H. 1993. Differential expression of the HMG box factors TCF-1 and LEF-1 during murine embryogenesis. Development 118: 439-448.

Pourquié, O. 2001. Vertebrate somitogenesis. Annu. Rev. Cell Dev. Biol. 17: $311-350$

. 2003. The segmentation clock: Converting embryonic time into spatial pattern. Science 301: 328-330.
Saga, Y. and Takeda, H. 2001. The making of the somite: Molecular events in vertebrate segmentation. Nat. Rev. Genet. 2: 835-845.

Saga, Y., Hata, N., Koseki, H., and Taketo, M.M. 1997. Mesp2: A novel mouse gene expressed in the presegmented mesoderm and essential for segmentation initiation. Genes \& Dev. 11: 1827-1839.

Takada, S., Stark, K.L., Shea, M.J., Vassileva, G., McMahon, J.A., and McMahon, A.P. 1994. Wnt-3a regulates somite and tailbud formation in the mouse embryo. Genes \& Dev. 8: 103-128.

Takahashi, Y., Inoue, T., Gossler, A., and Saga, Y. 2003. Feedback loops comprising DII1, DII3, and Mesp2, and differential involvement of Psen 1 are essential for rostrocaudal patterning of somites. Development 130: 4259-4268.

van Genderen, C., Okamura, R., Fariñas, I., Quo, R., Parslow, T., Bruhn, L., and Grosschedl, R. 1994. Development of several organs that require inductive epithelial mesenchymal interactions is impaired in LEF-1 deficient mice. Genes \& Dev. 8: 2691-2703.

van Noort, M. and Clevers, H. 2002. TCF transcription factors, mediators of Wnt-signaling in development and cancer. Dev. Biol. 244: 1-8.

Verbeek, S., Izon, D., Hofhuis, F., Robanus-Maandag, E., te Riele, H., van de Wetering, M., Oosterwegel, M., Wilson, A., MacDonald, H.R., and Clevers, H. 1995. An HMG-box-containing T-cell factor required for thymocyte differentiation. Nature 374: 70-74.

White, P.D., Farkas, D.R., McFadden, E.E., and Chapman, D.L. 2003. Defective somite patterning in mouse embryos with reduced levels of Tbx6. Development 120: 1681-1690.

Yamaguchi, T.P., Takada, S., Yoshikawa, Y., Wu, N., and McMahon, A.P. 1999. T (Brachyury) is a direct target of Wnt3a during paraxial mesoderm specification. Genes \& Dev. 13: 3185-3190.

Yoshikawa, Y., Fujimori, T., McMahon, A.P., and Takada, S. 1997. Evidence that absence of Wnt-3a signaling promotes neuralization instead of paraxial mesoderm development in the mouse. Dev. Biol. 183: $234-242$

Zhang, N. and Gridley, T. 1998. Defects in somite formation in lunatic fringe-deficient mice. Nature 394: 374-377. 


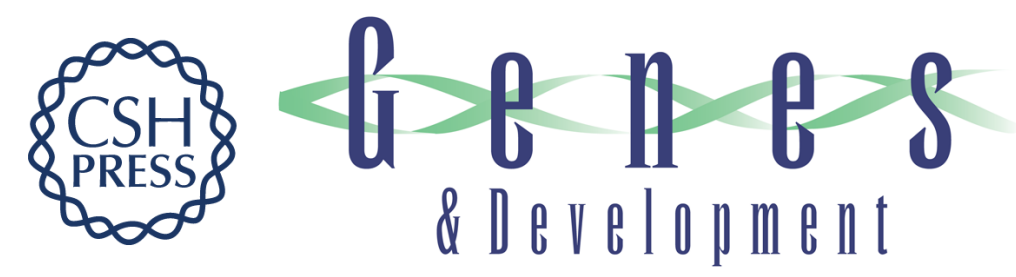

\section{LEF1-mediated regulation of Delta-like1 links Wnt and Notch signaling in somitogenesis}

Juan Galceran, Claudio Sustmann, Shu-Chi Hsu, et al.

Genes Dev. 2004, 18:

Access the most recent version at doi:10.1101/gad.1249504

\section{Supplemental http://genesdev.cshlp.org/content/suppl/2004/10/22/18.22.2718.DC1 \\ Material}

References This article cites 34 articles, 17 of which can be accessed free at: http://genesdev.cshlp.org/content/18/22/2718.full.html\#ref-list-1

\section{License}

Email Alerting Service

Receive free email alerts when new articles cite this article - sign up in the box at the top right corner of the article or click here.

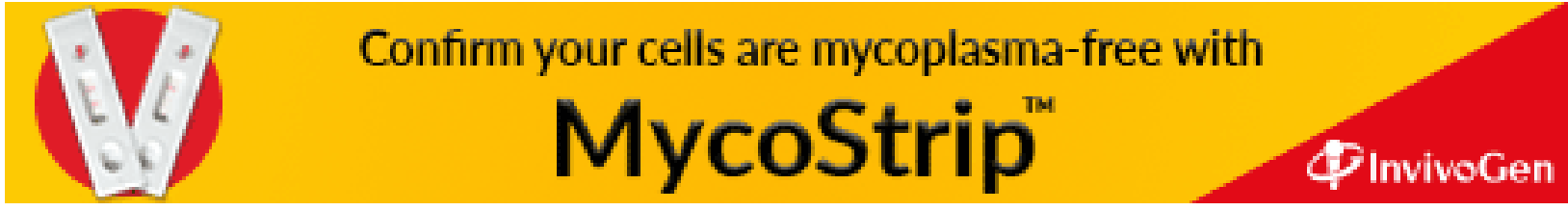

